\title{
Infinitely many solutions of degenerate quasilinear Schrödinger equation with general potentials
}

\author{
Yan Meng ${ }^{1} \mathbb{D}$, Xianjiu Huang ${ }^{1 *}$ and Jianhua Chen $^{1}$
}

\section{"Correspondence:}

xjhuangxwen@163.com

'Department of Mathematics,

Nanchang University, Nanchang,

Jiangxi 330031, P.R. China

\begin{abstract}
In this paper, we study the following quasilinear Schrödinger equation:

$$
-\operatorname{div}(a(x, \nabla u))+V(x)|x|^{-\alpha p^{*}}|u|^{p-2} u=K(x)|x|^{-\alpha p^{*}} f(x, u) \quad \text { in } \mathbb{R}^{N}
$$

where $N \geq 3,1<p<N,-\infty<\alpha<\frac{N-p}{p}, \alpha \leq e \leq \alpha+1, d=1+\alpha-e$,

$p^{*}:=p^{*}(\alpha, e)=\frac{N p}{N-d p}$ (critical Hardy-Sobolev exponent), $V$ and $K$ are nonnegative potentials, the function a satisfies suitable assumptions, and $f$ is superlinear, which is weaker than the Ambrosetti-Rabinowitz-type condition. By using variational methods we obtain that the quasilinear Schrödinger equation has infinitely many nontrivial solutions.
\end{abstract}

MSC: $35 \mathrm{~J} 60 ; 35 \mathrm{~J} 20$

Keywords: Schrödinger equation; Superlinear condition; Infinitely many solutions

\section{Introduction}

In this paper, we study the following quasilinear Schrödinger equation:

$$
\left\{\begin{array}{l}
-\operatorname{div}(a(x, \nabla u))+V(x)|x|^{-\alpha p^{*}}|u|^{p-2} u=K(x)|x|^{-\alpha p^{*}} f(x, u) \quad \text { in } \mathbb{R}^{N}, \\
u \in \mathcal{D}^{1, p}\left(\mathbb{R}^{N}\right) \cap \mathcal{D}_{\alpha}^{1, p}\left(\mathbb{R}^{N}\right)
\end{array}\right.
$$

where $N \geq 3,1<p<N,-\infty<\alpha<\frac{N-p}{p}, \alpha \leq e \leq \alpha+1, d=1+\alpha-e, p^{*}:=p^{*}(\alpha, e)=\frac{N p}{N-d p}$ is the critical Hardy-Sobolev exponent, $V$ and $K$ are nonnegative potentials, $f$ is of superlinear growth near infinity, and for some positive functions $h_{1}(x) \in L^{\infty}\left(\mathbb{R}^{N}\right)$ and $h_{0}(x) \in L_{\bar{\alpha}}^{p /(p-1)}\left(\mathbb{R}^{N}\right)$, where $\bar{\alpha}=\frac{\alpha p}{p^{*}}$, the function $a$ satisfies $|a(x, \nabla u)| \leq c_{0}|x|^{-\alpha p} h_{0}(x)+c_{0}(1+$ $\left.|x|^{-\alpha p}\right) h_{1}(x)|\nabla u|^{p-1}$.

Problem (1.1) comes from the quasilinear Schrödinger equation and involves several improvements. Firstly, Duc [17] established the existence of a nontrivial solution to the

(c) The Author(s) 2021. This article is licensed under a Creative Commons Attribution 4.0 International License, which permits use, sharing, adaptation, distribution and reproduction in any medium or format, as long as you give appropriate credit to the original author(s) and the source, provide a link to the Creative Commons licence, and indicate if changes were made. The images or other third party material in this article are included in the article's Creative Commons licence, unless indicated otherwise in a credit line to the material. If material is not included in the article's Creative Commons licence and your intended use is not permitted by statutory regulation or exceeds the permitted use, you will need to obtain permission directly from the copyright holder. To view a copy of this licence, visit http://creativecommons.org/licenses/by/4.0/. 
problem

$$
\left\{\begin{array}{l}
-\operatorname{div}(a(x, \nabla u))=f(x, u) \quad \text { in } \Omega, \\
u=0 \quad \text { on } \partial \Omega, u \in W_{0}^{1, p}(\Omega) .
\end{array}\right.
$$

For different types of $a(x, \nabla u)$, the quasilinear equation of the form (1.1) has been derived from several physical models. Especially, $a(x, \nabla u)=|\nabla u|^{p-2} \nabla u$ and $a(x, \nabla u)=$ $|x|^{-\alpha p}|\nabla u|^{p-2} \nabla u$ were used for the problems of nonlinear diffusion, such as nonlinear optics, plasma physics, condensed matter physics, and so on. We refer the reader to $[16,25]$ and references therein.

This type of equation has been extensively studied in recent years with a huge variety of hypotheses on the potentials $V(x)$ and $K(x)$. For $V$ bounded from below by a positive constant $\left(V(x) \geq V_{1}>0\right)$ and $K(x) \equiv 1$, we would like to cite $[1,10,21]$ and references therein, and in case of $K(x) \not \equiv 1$, we refer to [18, 23, 25].

If $V$ goes to zero as $|x| \rightarrow \infty$, that is,

$$
\lim _{|x| \rightarrow \infty} V(x)=0
$$

which is called the zero mass case, we can cite $[2,7,9]$, which use the same technique as that used in [2]. In the case where $K$ vanishes at infinity, we refer to the papers in [3-5]. The cases of $K$ bounded by a positive constant and unbounded $K$ are considered in [14].

Finally, $[11,12]$ deal with the comprehensive problems including the potentials $V$ and $K$. In [3], with more general potentials $K$ and $V$, the authors obtained an inequality of Hardy type and then the strong convergence in the whole space. As a matter of fact, they have obtained the compact embedding of $E \subset \mathcal{D}^{1,2}\left(\mathbb{R}^{N}\right)$ in $L_{K}^{q}\left(\mathbb{R}^{N}\right)$ with $2<q<2^{*}$. Using the same way, the compact embedding of $E \subset \mathcal{D}_{\alpha}^{1, p}\left(\mathbb{R}^{N}\right)$ in $L_{K, \alpha}^{q}\left(\mathbb{R}^{N}\right)$ is proved in [8] with $1<p<N, p<q<p^{*}$.

In most of the aforesaid references, the Ambrosetti-Rabinowitz (AR) condition is usually assumed. It is very crucial to ensure the boundedness of the Palais-Smale (PS) sequences of the energy functional. However, there are many functions that do not satisfy the AR condition. So in this paper, to prove that there are infinitely many solutions to quasilinear Schrödinger equation, we develop a superquadratic condition, which is weaker than the condition AR.

There are many difficulties in solving the problem of relationship among nonlinearities, operator, and potentials. To overcome this, we prove the existence of infinitely many solutions to problem (1.1) with compact embedding by using Tang's methods in [24]. As far as we know, to prove the boundedness of the $(C)_{c}$-sequence for problem (1.1), we must have compact embedding, so we need to enhance some conditions for potentials $K(x)$ and $V(x)$. Before proving our results, we need to make the following assumptions on $a, A, V$, $K$, and $f$.

(1) Functions $a$ and $A$. We consider continuous functions $a: \mathbb{R}^{N} \times \mathbb{R}^{N} \mapsto \mathbb{R}^{N}$ and $A: \mathbb{R}^{N} \times \mathbb{R}^{N} \mapsto \mathbb{R}$ such that $a(x, \xi)=\frac{\partial A(x, \xi)}{\partial \xi}$. Let $c_{0}$ and $c_{1}$ be positive real numbers, and let $h_{0}(x)$ and $h_{1}(x)$ be nonnegative measurable real functions in $\mathbb{R}^{N}$ such that $h_{0}(x) \in$ $L_{\bar{\alpha}}^{p /(p-1)}\left(\mathbb{R}^{N}\right)$ with $\bar{\alpha}=\frac{\alpha p}{p^{*}}$ and $h_{1}(x) \in L^{\infty}\left(\mathbb{R}^{N}\right)$ with $h_{1}(x) \geq 1$ for a.e. $x \in \mathbb{R}^{N}$. We introduce the following hypotheses:

$$
\left(A_{1}\right)|a(x, \xi)| \leq c_{0}|x|^{-\alpha p} h_{0}(x)+c_{0}\left(1+|x|^{-\alpha p}\right) h_{1}(x)|\xi|^{p-1} \text { for a.e. } x \in \mathbb{R}^{N} .
$$


$\left(A_{2}\right) \quad c_{1}\left(1+|x|^{-\alpha p}\right) h_{1}(x)|\xi-\eta|^{p} \leq(a(x, \xi)-a(x, \eta))(\xi-\eta)$ for all $\xi, \eta \in \mathbb{R}^{N}$ and a.e. $x \in \mathbb{R}^{N}$.

$\left(A_{3}\right) A$ is subhomogeneous, that is, $0 \leq a(x, \xi) \xi \leq p A(x, \xi)$ for all $\xi \in \mathbb{R}^{N}$ and a.e. $x \in \mathbb{R}^{N}$.

$\left(A_{4}\right) a(x, 0)=0, A(x, \xi)=A(x,-\xi)$ for a.e. $x \in \mathbb{R}^{N}$.

Remark 1.1 ([20]) The function $A$ can be used in several cases. For example:

(i) $A(x, \xi)=\frac{1}{p}|\xi|^{p}$.

(ii) $A(x, \xi)=\frac{h(x)}{p}|\xi|^{p}$ with $h \in L_{\mathrm{loc}}^{1}\left(\mathbb{R}^{N}\right)$.

(iii) $A(x, \xi)=\frac{1}{p}\left[\left(1+|\xi|^{2}\right)^{\frac{p}{2}}-1\right]$ with $p \geq 2$.

(iv) $A(x, \xi)=\frac{h(x)}{p}\left[\left(1+|\xi|^{2}\right)^{\frac{p}{2}}-1\right]$ with $p \geq 2$ and $h \in L^{\frac{p}{p-1}}\left(\mathbb{R}^{N}\right)$.

(v) $A(x, \xi)=\frac{1}{p}|\xi|^{p}+\theta(x)\left(\sqrt{1+|\xi|^{2}}-1\right)$ with a suitable function $\theta$. We get the operator $\operatorname{div}\left(|\nabla u|^{p-2} \nabla u\right)+\operatorname{div}\left(\theta(x) \frac{\nabla u}{\sqrt{1+|\nabla u|^{2}}}\right)$, which can be regarded as the sum of the $p$-Laplacian operator and a degenerate-form mean-curvature operator.

(2) Potentials $V$ and $K$.

$(V K 1) \quad V, K \in \mathcal{C}\left(\mathbb{R}^{N}, \mathbb{R}\right), V(x) \geq \min V(x) \geq 1, K(x) \geq \min K(x) \geq 0, K(x) \not \equiv 0$, and $K(x) \in L^{\infty}\left(\mathbb{R}^{N}\right)$

(VK2) $\lim _{|x| \rightarrow \infty} \frac{K(x)}{V^{\theta}(x)}=0$ for all $0<\theta<1$.

Example $1.2([15])$ The following functions are typical examples of functions satisfying $(V K 1)$ and $(V K 2)$ :

(i) $K(x)=2$ and $V(x)=(|x|+1)^{\frac{1}{\theta}}$ for $0<\theta<1$.

(ii) $K(x)=\sin x$ and $V(x)=[(|x|+1)(|\sin x|+1)]^{\frac{1}{\theta}}$ for $0<\theta<1$.

Is easy to check that $\lim _{|x| \rightarrow \infty} \frac{K(x)}{V^{\theta}(x)}=0, K(x) \not \equiv 0, K(x) \in L^{\infty}\left(\mathbb{R}^{N}\right), V(x) \geq \min V(x) \geq 1$, and $K(x) \geq \min K(x) \geq 0$ for all $0<\theta<1$.

(3) Functions $f$ and $F$. Let functions $f: \mathbb{R}^{N+1} \rightarrow \mathbb{R}$ and $F: \mathbb{R}^{N+1} \rightarrow \mathbb{R}$ such that $f(x, u)=$ $\frac{\partial F(x, u)}{\partial x}$ for all $x \in \mathbb{R}$ satisfy the following conditions:

$\left(f_{1}\right)$ there exist constants $c_{1}, c_{2}>0$ and $\beta \in\left(p, p^{*}\right)$ such that

$$
|f(x, u)| \leq c_{1}|u|^{p-1}+c_{2}|u|^{\beta-1} \quad \text { for all }(x, u) \in \mathbb{R}^{N} \times \mathbb{R} .
$$

$\left(f_{2}\right) \lim _{|u| \rightarrow \infty} \frac{|x|^{-\alpha p^{*}}|F(x, u)|}{|u|^{p}}=\infty$ for a.e. $x \in \mathbb{R}^{N}$, and there exists $r_{0} \geq 0$ such that

$$
F(x, u) \geq 0, \quad \forall(x, u) \in \mathbb{R}^{N} \times \mathbb{R},|u| \geq r_{0} .
$$

$\left(f_{3}\right) \mathcal{F}(x, u):=\frac{1}{p} u f(x, u)-F(x, u) \geq 0$, and there exist $c_{0}>0$ and $\kappa>\frac{N}{d p}$ such that

$$
|F(x, u)|^{\kappa} \leq c_{0}|u|^{p \kappa} \mathcal{F}(x, u), \quad \forall(x, u) \in \mathbb{R}^{N} \times \mathbb{R},|u| \geq r_{0} .
$$

$\left(f_{4}\right) f(x,-u)=-f(x, u), \forall(x, u) \in \mathbb{R}^{N} \times \mathbb{R}$.

$\left(f_{5}\right)$ There exist $\mu>p$ and $\varrho>0$ such that

$$
\mu F(x, u) \leq u f(x, u)+\varrho|u|^{p}, \quad \forall(x, u) \in \mathbb{R}^{N+1} .
$$

$\left(f_{6}\right)$ There exist $\mu>p$ and $r_{1}>0$ such that

$$
\mu F(x, u) \leq u f(x, u), \quad \forall(x, u) \in \mathbb{R}^{N+1},|u| \geq r_{1} .
$$


Example 1.3 ([19]) Is easy to check that the following nonlinearities $f$ satisfy $\left(f_{1}\right),\left(f_{2}\right),\left(f_{4}\right)$, and $\left(f_{6}\right)$ :

(i) $f(x, u)=g(x)|u|^{p-1} u\left[(p+3) u^{2}-2(p+2) u+p+1\right]$.

(ii) $f(x, u)=g(x)|u|^{p-2} u\left(4|u|^{3}+2 u \sin u-4 \cos u\right)$.

(iii) $f(x, u)=g(x) \sum_{i=1}^{m} b_{i}|u|^{\gamma_{i}} u$, where $b_{1}>0, b_{i} \in \mathbb{R}, i=2,3, \ldots, m$, $\gamma_{1}>\gamma_{2}>\cdots>\gamma_{m} \geq p-2, g \in \mathcal{C}\left(\mathbb{R}^{N}, \mathbb{R}\right)$, and $0<\inf _{\mathbb{R}^{N}} g \leq \sup _{\mathbb{R}^{N}} g<\infty$.

Now we are ready to state the main theorems of this paper.

Theorem 1.4 Let (VK1)-(VK2), $\left(A_{1}\right)-\left(A_{4}\right)$, and $\left(f_{1}\right)-\left(f_{4}\right)$ be satisfied. Then equation (1.1) has infinitely many nontrivial solutions.

Theorem 1.5 Let $(V K 1)-(V K 2),\left(A_{1}\right)-\left(A_{4}\right),\left(f_{1}\right),\left(f_{2}\right),\left(f_{4}\right)$, and $\left(f_{5}\right)$ be satisfied. The equation (1.1) has infinitely many nontrivial solutions.

It is easy to check that $\left(f_{1}\right)$ and $\left(f_{6}\right)$ imply $\left(f_{5}\right)$. Thus we have the following corollary.

Corollary 1.6 Let (VK1)-(VK2), $\left(A_{1}\right)-\left(A_{4}\right),\left(f_{1}\right),\left(f_{2}\right),\left(f_{4}\right)$, and $\left(f_{6}\right)$ be satisfied. Then equation (1.1) has infinitely many nontrivial solutions.

Remark 1.7 In our theorems, $F(x, u)$ is allowed to be sign-changing. Even if $F(x, u) \geq 0$, assumptions $\left(f_{2}\right),\left(f_{3}\right),\left(f_{5}\right)$, and $\left(f_{6}\right)$ seem to be weaker than the superquadratic conditions obtained in the aforementioned references.

Notations Considering $\alpha$ and $K$ in equation (1.1), an open set $B \subset \mathbb{R}$, and a measurable function $u: B \rightarrow \mathbb{R}$, we use the following notations.

- $L_{\alpha}^{q}(B)=\left\{u:\left.B \rightarrow \mathbb{R}\left|\int_{B}\right| x\right|^{-\alpha p^{*}}|u|^{q} \mathrm{~d} x<\infty\right\}$ for $1 \leq q<\infty$.

- $L_{K, \alpha}^{q}(B)=\left\{u:\left.B \rightarrow \mathbb{R}\left|\int_{B} K(x)\right| x\right|^{-\alpha p^{*}}|u|^{q} \mathrm{~d} x<\infty\right\}$ for $1 \leq q<\infty$.

- $\|u\|_{L_{K, \alpha}^{q}(B)}=\left(\int_{B} K(x)|x|^{-\alpha p^{*}}|u|^{q} \mathrm{~d} x\right)^{\frac{1}{q}}$ for $1 \leq q<\infty$.

- $\|u\|_{\mathcal{D}_{\alpha}^{1, p}(B)}=\left(\int_{B}|x|^{-\alpha p}|\nabla u|^{p} \mathrm{~d} x\right)^{\frac{1}{p}}$.

- $L^{q}(B)$ is the usual Sobolev space for $1 \leq q<\infty$.

- We denote by $o_{n}(1)$ terms that tend to zero as $n \rightarrow \infty$. The weak $(\rightarrow)$ and strong $(\rightarrow)$ convergences are always taken as $n \rightarrow \infty$.

- Hereafter $C$ is a positive constant that can changes its value in a sequence of inequalities.

The remainder of the paper is organized as follows. In Sect. 2, we present variational framework. In Sect. 3, we state and prove the main results of the paper.

\section{Variational framework}

In this section, we want to use variational methods. So we define a convenient space and functional. We consider the spaces

$$
\mathcal{D}^{1, p}\left(\mathbb{R}^{N}\right)=\left\{u: \mathbb{R}^{N} \rightarrow \mathbb{R} \mid u \in L^{p^{*}}\left(\mathbb{R}^{N}\right) \text { and } \nabla u \in L^{p}\left(\mathbb{R}^{N}\right)\right\}
$$

and

$$
\mathcal{D}_{\alpha}^{1, p}\left(\mathbb{R}^{N}\right)=\left\{u:\left.\mathbb{R}^{N} \rightarrow \mathbb{R}|| x\right|^{-\alpha} u \in L^{p^{*}}\left(\mathbb{R}^{N}\right) \text { and }|x|^{-\alpha} \nabla u \in L^{p}\left(\mathbb{R}^{N}\right)\right\} .
$$


We define

$$
E=\left\{\begin{array}{l|l}
u \in \mathcal{D}^{1, p}\left(\mathbb{R}^{N}\right) \cap \mathcal{D}_{\alpha}^{1, p}\left(\mathbb{R}^{N}\right) & \begin{array}{l}
\int_{\mathbb{R}^{N}}\left(1+|x|^{-\alpha p}\right)|\nabla u|^{p} \mathrm{~d} x<\infty \\
\text { and } \\
\int_{\mathbb{R}^{N}} V(x)|x|^{-\alpha p^{*}}|u|^{p} \mathrm{~d} x<\infty
\end{array}
\end{array}\right\},
$$

endowed with the norm

$$
\|u\|=\left(\int_{\mathbb{R}^{N}}\left(\left(1+|x|^{-\alpha p}\right)|\nabla u|^{p}+\frac{1}{k_{0} p} V(x)|x|^{-\alpha p^{*}}|u|^{p}\right) \mathrm{d} x\right)^{\frac{1}{p}}
$$

with $k_{0}$ given by the inequality $A(x, \nabla u) \geq k_{0} h_{1}(x)\left(1+|x|^{-\alpha p}\right)|\nabla u|^{p}$ for all $\xi \in \mathbb{R}^{N}$ and a.e. $x \in \mathbb{R}^{N}$, which will be proved in Lemma 3.2. Evidently, $E$ is continuously embedded into $\mathcal{D}_{\alpha}^{1, p}\left(\mathbb{R}^{N}\right)$. By the weighted Caffarelli-Kohn-Nirenberg's inequality [13]

$$
\left(\int_{\mathbb{R}^{N}}|x|^{-\alpha p^{*}}|u|^{p^{*}} \mathrm{~d} x\right)^{\frac{1}{p^{*}}} \leq C\left(\int_{\mathbb{R}^{N}}|x|^{-\alpha p}|\nabla u|^{p} \mathrm{~d} x\right)^{\frac{1}{p}},
$$

$\mathcal{D}_{\alpha}^{1, p}\left(\mathbb{R}^{N}\right)$ is continuously embedded into $L_{\alpha}^{p^{*}}\left(\mathbb{R}^{N}\right)$. Thus we get $E \hookrightarrow \mathcal{D}_{\alpha}^{1, p}\left(\mathbb{R}^{N}\right) \hookrightarrow L_{\alpha}^{p^{*}}\left(\mathbb{R}^{N}\right)$ for $N \geq 3$.

In $E$, we define the following energy functional $J \in \mathcal{C}^{1}(E, \mathbb{R})$ :

$$
\begin{aligned}
J(u)= & \int_{\mathbb{R}^{N}}\left(A(x, \nabla u)+\frac{1}{p} V(x)|x|^{-\alpha p^{*}}|u|^{p}\right) \mathrm{d} x \\
& -\int_{\mathbb{R}^{N}} K(x)|x|^{-\alpha p^{*}} F(x, u) \mathrm{d} x, \quad \forall u \in E .
\end{aligned}
$$

Its Gateaux derivative is given by

$$
\begin{aligned}
\left\langle J^{\prime}(u), v\right\rangle= & \int_{\mathbb{R}^{N}}\left(a(x, \nabla u) \nabla v+V(x)|x|^{-\alpha p^{*}}|u|^{p-2} u v\right) \mathrm{d} x \\
& -\int_{\mathbb{R}^{N}} K(x)|x|^{-\alpha p^{*}} f(x, u) v \mathrm{~d} x, \quad \forall u, v \in E .
\end{aligned}
$$

By condition $\left(f_{1}\right)$ we have

$$
|F(x, u)| \leq \frac{c_{1}}{p}|u|^{p}+\frac{c_{2}}{\beta}|u|^{\beta}, \quad \forall(x, u) \in \mathbb{R}^{N} \times \mathbb{R} .
$$

\section{Existence of infinitely many solutions}

In this section, we prove the existence of infinitely many solutions for problem (1.1). Next, we give the definition of a $(C)_{c}$-sequence.

A sequence $\left\{u_{n}\right\} \subset X$ is said to be a $(C)_{c}$-sequence if $J\left(u_{n}\right) \rightarrow c$ and $\left\|J^{\prime}\left(u_{n}\right)\right\|\left(1+\left\|u_{n}\right\|\right) \rightarrow$ 0 , and it is said to satisfy the $(C)_{c}$-condition if any $(C)_{c}$-sequence has a convergent subsequence.

To prove our results, we use the following symmetric mountain pass theorem.

Lemma $3.1([6,22])$ Let $X$ be an infinite-dimensional Banach space, $X=Y \oplus Z$, where $Y$ is finite dimensional. Suppose that $I \in \mathcal{C}^{1}(X, \mathbb{R})$ satisfies the $(C)_{c}$-condition for all $c>0$ and the following conditions: 
(I $\left.I_{1}\right) I(0)=0, I(-u)=I(u)$ for all $u \in X$;

$\left(I_{2}\right)$ there exist constants $\rho, \theta>0$ such that $\left.I\right|_{\partial B_{\rho} \cap Z} \geq \theta$;

$\left(I_{3}\right)$ for any finite-dimensional subspace $\tilde{X} \subset X$, there is $R=R(\tilde{X})>0$ such that $I(u) \leq 0$ on $\tilde{X} \backslash B_{R}$.

Then I possesses an unbounded sequence of critical values.

Lemma 3.2 ([20]) The function A satisfies

$$
|A(x, \xi)| \leq c_{0}|x|^{-\alpha p}\left(h_{0}(x)|\xi|+h_{1}(x)|\xi|^{p}\right)+c_{0} h_{1}(x)|\xi|^{p}, \quad \text { a.e. } x \in \mathbb{R}^{N},
$$

and there exists $k_{0}>0$ such that

$$
A(x, \xi) \geq k_{0} h_{1}(x)\left(1+|x|^{-\alpha p}\right)|\xi|^{p} \quad \text { for all } \xi \in \mathbb{R}^{N} \text { and a.e. } x \in \mathbb{R}^{N} \text {. }
$$

The following two lemmas discuss the continuous and compact embedding $E \hookrightarrow$ $L_{K, \alpha}^{q}\left(\mathbb{R}^{N}\right)$ for all $q \in\left[p, p^{*}\right)$.

Lemma 3.3 Let (VK1)-(VK2) be satisfied. Then $E$ is continuously embedded in $L_{K, \alpha}^{q}\left(\mathbb{R}^{N}\right)$ for all $q \in\left[p, p^{*}\right)$, that is, there exists $\gamma_{q}>0$ such that

$$
\|u\|_{L_{K, \alpha}^{q}\left(\mathbb{R}^{N}\right)} \leq \gamma_{q}\|u\|, \quad \forall u \in E
$$

Proof Since $\frac{K(x)}{V^{\theta}(x)} \rightarrow 0$ as $|x| \rightarrow \infty$ and $0<\frac{K(x)}{V(x)} \leq \frac{K(x)}{V^{\theta}(x)}$, we have $\frac{K(x)}{V(x)} \rightarrow 0$ as $|x| \rightarrow \infty$. By the continuity of $V(x)$ and $K(x)$ there exists $M>0$ such that $K(x) \leq M V^{\theta}(x) \leq M V(x)$ for all $x \in \mathbb{R}^{N}$ and $0<\theta<1$. If $q=p$, then the proof is trivial. Fix $q \in\left(p, p^{*}\right)$ and choose $\sigma=\frac{p^{*}-q}{p^{*}-p}$. Then $q=p \sigma+(1-\sigma) p^{*}$ and $0<\sigma<1$. From $\mathcal{D}_{\alpha}^{1, p}\left(\mathbb{R}^{N}\right) \hookrightarrow L_{\alpha}^{p^{*}}\left(\mathbb{R}^{N}\right),(V K 2),(2.1)$, and Hölder's inequality we can get the following inequality:

$$
\begin{aligned}
\|u\|_{K, \alpha}^{q} & =\int_{\mathbb{R}^{N}} K(x)|x|^{-\alpha p^{*}}|u|^{q} \mathrm{~d} x \\
& =\int_{\mathbb{R}^{N}} K(x)|x|^{-\alpha p^{*}}|u|^{p \sigma}|u|^{(1-\sigma) p^{*}} \mathrm{~d} x \\
& \leq\left[\int_{\mathbb{R}^{N}} K(x)^{\frac{1}{\sigma}}|x|^{-\alpha p^{*}}|u|^{p}\right]^{\sigma}\left[\int_{\mathbb{R}^{N}}|x|^{-\alpha p^{*}}|u|^{p^{*}} \mathrm{~d} x\right]^{1-\sigma} \\
& \leq\left(\sup _{x \in \mathbb{R}^{N}} \frac{K(x)}{|V(x)|^{\sigma}}\right)\left(\int_{\mathbb{R}^{N}} V(x)|x|^{-\alpha p^{*}}|u|^{p}\right)^{\sigma}\left(\int_{\mathbb{R}^{N}}|x|^{-\alpha p^{*}}|u|^{p^{*}} \mathrm{~d} x\right)^{1-\sigma} \\
& \leq C M\left(\int_{\mathbb{R}^{N}} V(x)|x|^{-\alpha p^{*}}|u|^{p}\right)^{\sigma}\left(\int_{\mathbb{R}^{N}}|x|^{-\alpha p}|\nabla u|^{p} \mathrm{~d} x\right)^{\frac{p^{*}(1-\sigma)}{p}} \\
& \leq p k_{0} C M\left(\int_{\mathbb{R}^{N}}\left(\left(1+|x|^{-\alpha p}\right)|\nabla u|^{p}+\frac{1}{p k_{0}} V(x)|x|^{-\alpha p^{*}}|u|^{p}\right) \mathrm{d} x\right)^{\sigma+\frac{p^{*}(1-\sigma)}{p}} \\
& =p k_{0} C M\left(\int_{\mathbb{R}^{N}}\left(\left(1+|x|^{-\alpha p}\right)|\nabla u|^{p}+\frac{1}{p k_{0}} V(x)|x|^{-\alpha p^{*}}|u|^{p}\right) \mathrm{d} x\right)^{\frac{q}{p}} \\
& =p k_{0} C M\|u\|^{q} .
\end{aligned}
$$

It follows that $E \hookrightarrow L_{K, \alpha}^{q}\left(\mathbb{R}^{N}\right)$ is a continuous embedding. 
Lemma 3.4 Let (VK1)-(VK2) be satisfied. Then $E$ is compactly embedded in $L_{K, \alpha}^{q}\left(\mathbb{R}^{N}\right)$ for all $q \in\left[p, p^{*}\right)$.

Proof From Lemma 3.3 we have $\frac{K(x)}{V(x)} \rightarrow 0$ as $|x| \rightarrow \infty$. Hence for any $\varepsilon>0$, there exists $R>0$ such that $K(x) \leq \varepsilon V(x)$ for $|x|>R$. Let $\left\{u_{n}\right\} \subset E$ be a bounded sequence of $E$. Going if necessary to a subsequence, we may assume that

$$
u_{n} \rightarrow 0 \quad \text { in } E, \quad u_{n} \rightarrow 0 \quad \text { in } L_{K, \alpha, \text { loc }}^{q}\left(\mathbb{R}^{N}\right) \quad \text { for } p \leq q<p^{*} .
$$

Next, we claim that $u_{n} \rightarrow 0$ in $L_{K, \alpha}^{p}\left(\mathbb{R}^{N}\right)$. Set $B_{R}(0)=\left\{x \in \mathbb{R}^{N}:|x| \leq R\right\}$. Then

$$
\begin{aligned}
\int_{\mathbb{R}^{N} \backslash B_{R}} K(x)|x|^{-\alpha p^{*}}\left|u_{n}(x)\right|^{p} \mathrm{~d} x & <\varepsilon \int_{\mathbb{R}^{N}} V(x)|x|^{-\alpha p^{*}}\left|u_{n}(x)\right|^{p} \mathrm{~d} x \\
& \leq p k_{0} \varepsilon\left\|u_{n}\right\|^{p} .
\end{aligned}
$$

Hence, for any $\varepsilon>0$, we have

$$
\begin{aligned}
& \int_{\mathbb{R}^{N}} K(x)|x|^{-\alpha p^{*}}\left|u_{n}(x)\right|^{p} \mathrm{~d} x \\
& \quad=\int_{B_{R}} K(x)|x|^{-\alpha p^{*}}\left|u_{n}(x)\right|^{p} \mathrm{~d} x+\int_{\mathbb{R}^{N} \backslash B_{R}} K(x)|x|^{-\alpha p^{*}}\left|u_{n}(x)\right|^{p} \mathrm{~d} x \\
& \quad<\varepsilon\left(1+p k_{0}\left\|u_{n}\right\|^{p}\right),
\end{aligned}
$$

from which (3.4) follows. Since $|s|^{q} /|s|^{p} \rightarrow 0$ as $s \rightarrow 0$ and $|s|^{q} /|s|^{p^{*}} \rightarrow 0$ as $s \rightarrow \infty$, then for any $\varepsilon>0$, there exists $C>0$ such that

$$
K(x)|s|^{q} \leq \varepsilon C K(x)\left(|s|^{p}+|s|^{p^{*}}\right)+C K(x)|s|^{p} \quad \text { for all } s \in \mathbb{R} .
$$

To prove the lemma for general exponent $q$, we use an interpolation argument. Let $u_{n} \rightarrow$ 0 in $E$. We have just proved that $u_{n} \rightarrow 0$ in $L_{K, \alpha}^{q}\left(\mathbb{R}^{N}\right)$, that is,

$$
\int_{\mathbb{R}^{N}} K(x)|x|^{-\alpha p^{*}}\left|u_{n}(x)\right|^{q} \mathrm{~d} x \rightarrow 0
$$

Since the embedding $E \hookrightarrow L_{\alpha}^{p^{*}}\left(\mathbb{R}^{N}\right)$ is continuous and $\left\{u_{n}\right\}$ is bounded in $E$, we also have that $\left\{u_{n}\right\}$ is bounded in $L_{\alpha}^{p^{*}}\left(\mathbb{R}^{N}\right)$. From (3.5) we have

$$
\begin{aligned}
\int_{\mathbb{R}^{N}} K(x)|x|^{-\alpha p^{*}}\left|u_{n}(x)\right|^{q} \mathrm{~d} x \leq & \varepsilon C \int_{\mathbb{R}^{N}}\left(K(x)|x|^{-\alpha p^{*}}\left|u_{n}\right|^{p}+|x|^{-\alpha p^{*}}\left|u_{n}\right|^{p^{*}}\right) \mathrm{d} x \\
& +C \int_{\mathbb{R}^{N}} K(x)|x|^{-\alpha p^{*}}\left|u_{n}\right|^{p} \mathrm{~d} x \rightarrow 0,
\end{aligned}
$$

implying that $u_{n} \rightarrow 0$ in $L_{K, \alpha}^{q}\left(\mathbb{R}^{N}\right)$. This completes the proof.

Next, we need the following lemmas to show that $J$ satisfies Lemma 3.1 . 
Lemma 3.5 Under assumptions (VK1), $(V K 2),\left(f_{1}\right),\left(f_{2}\right)$, and $\left(f_{3}\right)$, any sequence $\left\{u_{n}\right\} \subset E$ satisfying

$$
J\left(u_{n}\right) \rightarrow c^{*}>0, \quad\left\langle J^{\prime}\left(u_{n}\right), u_{n}\right\rangle \rightarrow 0,
$$

is bounded in $E$.

Proof To prove the boundedness of $\left\{u_{n}\right\}$, arguing by contradiction, suppose that $\left\|u_{n}\right\| \rightarrow$ $\infty$ as $n \rightarrow \infty$. Let $v_{n}=\frac{u_{n}}{\left\|u_{n}\right\|}$. Then $\left\|v_{n}\right\|=1$. Observe that for large $n$,

$$
\begin{aligned}
c^{*}+1 \geq & J\left(u_{n}\right)-\frac{1}{p}\left\langle J^{\prime}\left(u_{n}\right), u_{n}\right\rangle \\
= & \int_{\mathbb{R}^{N}}\left[A\left(x, \nabla u_{n}\right)-\frac{1}{p} a\left(x, \nabla u_{n}\right) \nabla u_{n}\right] \mathrm{d} x \\
& +\int_{\mathbb{R}^{N}} K(x)|x|^{-\alpha p^{*}}\left[\frac{1}{p} f\left(x, u_{n}\right) u_{n}-F\left(x, u_{n}\right)\right] \mathrm{d} x \\
\geq & \int_{\mathbb{R}^{N}} K(x)|x|^{-\alpha p^{*}}\left[\frac{1}{p} f\left(x, u_{n}\right) u_{n}-F\left(x, u_{n}\right)\right] \mathrm{d} x \\
= & \int_{\mathbb{R}^{N}} K(x)|x|^{-\alpha p^{*}} \mathcal{F}\left(x, u_{n}\right) \mathrm{d} x .
\end{aligned}
$$

It follows from (2.2) and (3.2) that

$$
\begin{aligned}
\int_{\mathbb{R}^{N}} \frac{K(x)|x|^{-\alpha p^{*}}\left|F\left(x, u_{n}\right)\right|}{\left\|u_{n}\right\|^{p}} \mathrm{~d} x \\
\quad \geq \frac{\int_{\mathbb{R}^{N}}\left(A\left(x, \nabla u_{n}\right)+\frac{1}{p} V(x)|x|^{-\alpha p^{*}}\left|u_{n}\right|^{p}\right) \mathrm{d} x-J\left(u_{n}\right)}{\left\|u_{n}\right\|^{p}} \\
\geq \frac{\int_{\mathbb{R}^{N}}\left(k_{0} h_{1}(x)\left(1+|x|^{-\alpha p}\right)\left|\nabla u_{n}\right|^{p}+\frac{1}{p} V(x)|x|^{-\alpha p^{*}}\left|u_{n}\right|^{p}\right) \mathrm{d} x-J\left(u_{n}\right)}{\left\|u_{n}\right\|^{p}} \\
\quad \geq \frac{k_{0}\left\|u_{n}\right\|^{p}-J\left(u_{n}\right)}{\left\|u_{n}\right\|^{p}} .
\end{aligned}
$$

By (3.8) we obtain

$$
0<k_{0} \leq \limsup _{n \rightarrow \infty} \int_{\mathbb{R}^{N}} \frac{K(x)|x|^{-\alpha p^{*}}\left|F\left(x, u_{n}\right)\right|}{\left\|u_{n}\right\|^{p}} \mathrm{~d} x .
$$

For $0 \leq a<b$, let

$$
\Omega_{n}(a, b)=\left\{x \in \mathbb{R}^{N}: a \leq\left|u_{n}(x)\right|<b\right\} .
$$

Passing to a subsequence, we may assume that $v_{n} \rightarrow v$ in $E$ is satisfied. Then by Lemma 3.4, $E$ is compactly embedded in $L_{K, \alpha}^{q}\left(\mathbb{R}^{N}\right), q \in\left[p, p^{*}\right), v_{n} \rightarrow v$ in $L_{K, \alpha}^{q}\left(\mathbb{R}^{N}\right), q \in\left[p, p^{*}\right)$, and $v_{n} \rightarrow v$ a.e. on $\mathbb{R}^{N}$. 
If $v=0$, then $v_{n} \rightarrow 0$ in $L_{K, \alpha}^{q}\left(\Omega_{n}\right), q \in\left[p, p^{*}\right)$, and $v_{n} \rightarrow 0$ a.e. on $\mathbb{R}^{N}$. Hence from (2.4) it follows that

$$
\begin{aligned}
& \int_{\Omega_{n}\left(0, r_{0}\right)} \frac{K(x)|x|^{-\alpha p^{*}}\left|F\left(x, u_{n}\right)\right|}{\left|u_{n}\right|^{p}}\left|v_{n}\right|^{p} \mathrm{~d} x \\
& \leq\left(\frac{c_{1}}{p}+\frac{c_{2}}{\beta} r_{0}^{\beta-p}\right) \int_{\Omega_{n}\left(0, r_{0}\right)} K(x)|x|^{-\alpha p^{*}}\left|v_{n}\right|^{p} \mathrm{~d} x \\
& =\left(\frac{c_{1}}{p}+\frac{c_{2}}{\beta} r_{0}^{\beta-p}\right)\left\|v_{n}\right\|_{L_{K, \alpha}^{p}\left(\Omega_{n}\left(0, r_{0}\right)\right)}^{p} \rightarrow 0 .
\end{aligned}
$$

Set $\kappa^{\prime}=\frac{\kappa}{\kappa-1}$. Since $\kappa>\frac{N}{d p}$, we see that $p \kappa^{\prime} \in\left(p, p^{*}\right)$. Hence from $\left(f_{3}\right)$ and (3.7) we have

$$
\begin{aligned}
& \int_{\Omega_{n}\left(r_{0}, \infty\right)} \frac{K(x)|x|^{-\alpha p^{*}}\left|F\left(x, u_{n}\right)\right|}{\left|u_{n}\right|^{p}}\left|v_{n}\right|^{p} \mathrm{~d} x \\
& \leq\left[\int_{\Omega_{n}\left(r_{0}, \infty\right)} \frac{K(x)|x|^{-\alpha p^{*}}\left|F\left(x, u_{n}\right)\right|^{\kappa}}{\left|u_{n}\right|^{p \kappa}} \mathrm{d} x\right]^{\frac{1}{\kappa}}\left[\int_{\Omega_{n}\left(r_{0}, \infty\right)} K(x)|x|^{-\alpha p^{*}}\left|v_{n}\right|^{p \kappa^{\prime}} \mathrm{d} x\right]^{\frac{1}{\kappa^{\prime}}} \\
& \leq\left[\int_{\Omega_{n}\left(r_{0}, \infty\right)} \frac{K(x)|x|^{-\alpha p^{*}} c_{0}\left|u_{n}\right|^{p \kappa} \mathcal{F}\left(x, u_{n}\right)}{\left|u_{n}\right|^{p \kappa}} \mathrm{d} x\right]^{\frac{1}{\kappa}}\left\|v_{n}\right\|_{L_{K, \alpha}^{p \kappa^{\prime}}\left(\Omega_{n}\left(r_{0}, \infty\right)\right)}^{p} \\
& \leq\left[c_{0}\left(c^{*}+1\right)\right]^{\frac{1}{\kappa}}\left\|v_{n}\right\|_{L_{K, \alpha}^{p \kappa^{\prime}}\left(\mathbb{R}^{N}\right)}^{p} \rightarrow 0 .
\end{aligned}
$$

Combining (3.11) with (3.12), we have

$$
\int_{\mathbb{R}^{N}} \frac{K(x)|x|^{-\alpha p^{*}}\left|F\left(x, u_{n}\right)\right|}{\left\|u_{n}\right\|^{p}} \mathrm{~d} x \rightarrow 0,
$$

which contradicts (3.9).

Set $A:=\left\{x \in \mathbb{R}^{N}: v(x) \neq 0\right\}$. If $v \neq 0$, then meas $(A)>0$. For a.e. $x \in A$, we have $\lim _{n \rightarrow \infty}\left|u_{n}(x)\right|=\infty$. Hence $A \subset \Omega_{n}\left(r_{0}, \infty\right)$ for large $n \in \mathbb{N}$, and from Hölder's inequality, $h_{0}(x) \in L_{\bar{\alpha}}^{p /(p-1)}\left(\mathbb{R}^{N}\right), h_{1}(x) \in L^{\infty}\left(\mathbb{R}^{N}\right), h_{1}(x) \geq 1,(2.2),(2.4),(3.1),\left(f_{2}\right)$, and Fatou's lemma it follows that

$$
\begin{aligned}
0= & \lim _{n \rightarrow \infty} \frac{c^{*}+o_{n}(1)}{\left\|u_{n}\right\|^{p}}=\lim _{n \rightarrow \infty} \frac{J\left(u_{n}\right)}{\left\|u_{n}\right\|^{p}} \\
= & \lim _{n \rightarrow \infty} \frac{\int_{\mathbb{R}^{N}}\left(A\left(x, \nabla u_{n}\right)+\frac{1}{p} V(x)|x|^{-\alpha p^{*}}\left|u_{n}\right|^{p}\right) \mathrm{d} x-\int_{\mathbb{R}^{N}} K(x)|x|^{-\alpha p^{*}} F\left(x, u_{n}\right) \mathrm{d} x}{\left\|u_{n}\right\|^{p}} \\
\leq & \limsup _{n \rightarrow \infty} \frac{\int_{\mathbb{R}^{N}}\left[c_{0} h_{1}(x)\left(1+|x|^{-\alpha p}\right)\left|\nabla u_{n}\right|^{p}+\frac{1}{p} V(x)|x|^{-\alpha p^{*}}\left|u_{n}\right|^{p}\right] \mathrm{d} x}{\left\|u_{n}\right\|^{p}} \\
& +\limsup _{n \rightarrow \infty} \frac{\int_{\mathbb{R}^{N}} c_{0}|x|^{-\alpha p} h_{0}(x)\left|\nabla u_{n}\right| \mathrm{d} x}{\left\|u_{n}\right\|^{p}}-\liminf _{n \rightarrow \infty} \frac{\int_{\mathbb{R}^{N}} K(x)|x|^{-\alpha p^{*}} F\left(x, u_{n}\right) \mathrm{d} x}{\left\|u_{n}\right\|^{p}} \\
\leq & \limsup _{n \rightarrow \infty} \frac{\max \left\{k_{0}, c_{0} h_{1}(x)\right\}\left\|u_{n}\right\|^{p}}{\left\|u_{n}\right\|^{p}} \\
& +\limsup _{n \rightarrow \infty} \frac{c_{0}\left[\int_{\mathbb{R}^{N}}|x|^{-\alpha p} h_{0}(x)^{\frac{p}{p-1}} \mathrm{~d} x\right]^{\frac{p-1}{p}}\left[\int_{\mathbb{R}^{N}}|x|^{-\alpha p}\left|\nabla u_{n}\right|^{p} \mathrm{~d} x\right]^{\frac{1}{p}}}{\left\|u_{n}\right\|^{p}}
\end{aligned}
$$




$$
\begin{aligned}
& -\liminf _{n \rightarrow \infty} \frac{\int_{\mathbb{R}^{N}} K(x)|x|^{-\alpha p^{*}} F\left(x, u_{n}\right) \mathrm{d} x}{\left\|u_{n}\right\|^{p}} \\
\leq & \max \left\{k_{0}, c_{0} h_{1}(x)\right\}+\limsup _{n \rightarrow \infty} \frac{c_{0}\left\|h_{0}\right\|_{L_{\alpha}^{p(p-1)}\left(\mathbb{R}^{N}\right)}\left\|u_{n}\right\|}{\left\|u_{n}\right\|^{p}} \\
& +\limsup _{n \rightarrow \infty}\left[\int_{\Omega_{n}\left(0, r_{0}\right)} \frac{K(x)|x|^{-\alpha p^{*}} F\left(x, u_{n}\right)}{\left|u_{n}\right|^{p}}\left|v_{n}\right|^{p} \mathrm{~d} x\right] \\
& -\liminf _{n \rightarrow \infty}\left[\int_{\Omega_{n}\left(r_{0}, \infty\right)} \frac{K(x)|x|^{-\alpha p^{*}} F\left(x, u_{n}\right)}{\left|u_{n}\right|^{p}}\left|v_{n}\right|^{p} \mathrm{~d} x\right] \\
= & \max \left\{k_{0}, c_{0} h_{1}(x)\right\}+\limsup _{n \rightarrow \infty}\left[\left(\frac{c_{1}}{p}+\frac{c_{2}}{\beta} r_{0}^{\beta-p}\right)\left\|v_{n}\right\|_{L_{K, \alpha}^{p}\left(\Omega_{n}\left(0, r_{0}\right)\right)}^{p}\right] \\
& -\liminf _{n \rightarrow \infty}\left[\int_{\Omega_{n}\left(r_{0}, \infty\right)} \frac{K(x)|x|^{-\alpha p^{*}}\left|F\left(x, u_{n}\right)\right|}{\left|u_{n}\right|^{p}}\left[\chi_{\Omega_{n}\left(r_{0}, \infty\right)}(x)\right]\left|v_{n}\right|^{p} \mathrm{~d} x\right] \\
\leq & \max \left\{k_{0}, c_{0} h_{1}(x)\right\}+\left(\frac{c_{1}}{p}+\frac{c_{2}}{\beta} r_{0}^{\beta-p}\right) \gamma_{p}^{p} \\
& -\liminf _{n \rightarrow \infty}\left[\int_{\Omega_{n}\left(r_{0}, \infty\right)} \frac{K(x)|x|^{-\alpha p^{*}}\left|F\left(x, u_{n}\right)\right|}{\left|u_{n}\right|^{p}}\left[\chi_{\Omega_{n}\left(r_{0}, \infty\right)}(x)\right]\left|v_{n}\right|^{p} \mathrm{~d} x\right] \\
= & -\infty, \quad
\end{aligned}
$$

which is a contradiction. Thus $\left\{u_{n}\right\}$ is bounded in $E$.

Lemma 3.6 Let $p_{1}, p_{2}>1, r, q \geq 1$, and $\Omega \subseteq \mathbb{R}^{N}$. Let $g(x, t)$ be a Carathéodory function on $\Omega \times \mathbb{R}$ satisfying

$$
|g(x, t)| \leq a_{1}|t|^{\left(p_{1}-1\right) / r}+a_{2}|t|^{\left(p_{2}-1\right) / r}, \quad \forall(x, t) \in \Omega \times \mathbb{R}
$$

where $a_{1}, a_{2} \geq 0$. If $u_{n} \rightarrow u$ in $L_{K, \alpha}^{p_{1}}(\Omega) \cap L_{K, \alpha}^{p_{2}}(\Omega)$ and $u_{n} \rightarrow u$ for a.e. $x \in \Omega$. Then for any $v \in L_{K, \alpha}^{p_{1} q}(\Omega) \cap L_{K, \alpha}^{p_{2} q}(\Omega)$,

$$
\lim _{n \rightarrow \infty} \int_{\Omega} K(x)|x|^{-\alpha p^{*}}\left|g\left(x, u_{n}\right)-g(x, u)\right|^{r}|v|^{q} d x=0 .
$$

Proof If (3.15) is not true, then there exist a constant $\varepsilon_{0}>0$ and a subsequence $\left\{u_{k_{i}}\right\}$ such that

$$
\int_{\Omega} K(x)|x|^{-\alpha p^{*}}\left|g\left(x, u_{k_{i}}\right)-g(x, u)\right|^{r}|v|^{q} \mathrm{~d} x \geq \varepsilon_{0}, \quad \forall i \in \mathbb{N} .
$$

Since $u_{n} \rightarrow u$ in $L_{K, \alpha}^{p_{1}}(\Omega) \cap L_{K, \alpha}^{p_{2}}(\Omega)$, passing to a subsequence if necessary, we can assume that $\sum_{i=1}^{\infty}\left\|u_{k_{i}}-u\right\|_{L_{K, \alpha}^{p_{1}}}^{p_{1}}<+\infty$ and $\sum_{i=1}^{\infty}\left\|u_{k_{i}}-u\right\|_{L_{K, \alpha}^{p_{2}}}^{p_{2}}<+\infty$. Set

$$
w_{1}(x)=\left[\sum_{i=1}^{\infty}\left|u_{k_{i}}-u\right|^{p_{1}}\right]^{\frac{1}{p_{1}}}, \quad w_{2}(x)=\left[\sum_{i=1}^{\infty}\left|u_{k_{i}}-u\right|^{p_{2}}\right]^{\frac{1}{p_{2}}}, \quad x \in \Omega .
$$


Then $w_{1} \in L_{K, \alpha}^{p_{1}}(\Omega)$ and $w_{2} \in L_{K, \alpha}^{p_{2}}(\Omega)$. Note that

$$
\begin{aligned}
K(x)|x|^{-\alpha p^{*}}\left|g\left(x, u_{k_{i}}\right)-g(x, u)\right|^{r}|v|^{q} \\
\leq 2^{r-1} K(x)|x|^{-\alpha p^{*}}\left(\left|g\left(x, u_{k_{i}}\right)\right|^{r}+|g(x, u)|^{r}\right)|v|^{q} \\
\leq 4^{r-1} K(x)|x|^{-\alpha p^{*}}\left[a_{1}^{r}\left(\left|u_{k_{i}}\right|^{p_{1}-1}+|u|^{p_{1}-1}\right)+a_{2}^{r}\left(\left|u_{k_{i}}\right|^{p_{2}-1}+|u|^{p_{2}-1}\right)\right]|v|^{q} \\
\leq 4^{r-1} K(x)|x|^{-\alpha p^{*}} \\
\quad \times\left[2^{p_{1}+1} a_{1}^{r}\left(\left|u_{k_{i}}-u\right|^{p_{1}-1}+|u|^{p_{1}-1}\right)+2^{p_{2}+1} a_{2}^{r}\left(\left|u_{k_{i}}-u\right|^{p_{2}-1}+|u|^{p_{2}-1}\right)\right]|v|^{q} \\
\leq 4^{r-1} K(x)|x|^{-\alpha p^{*}}\left[2^{p_{1}+1} a_{1}^{r}\left(\left|w_{1}\right|^{p_{1}-1}+|u|^{p_{1}-1}\right)+2^{p_{2}+1} a_{2}^{r}\left(\left|w_{2}\right|^{p_{2}-1}+|u|^{p_{2}-1}\right)\right]|v|^{q} \\
:=h(x), \quad \forall i \in \mathbb{N}, x \in \Omega,
\end{aligned}
$$

and

$$
\begin{aligned}
\int_{\Omega} h(x) \mathrm{d} x= & 4^{r-1}\left[2^{p_{1}+1} a_{1}^{r} \int_{\Omega} K(x)|x|^{-\alpha p^{*}}\left(\left|w_{1}\right|^{p_{1}-1}+|u|^{p_{1}-1}\right)|v|^{q} \mathrm{~d} x\right. \\
& \left.+2^{p_{2}+1} a_{2}^{r} \int_{\Omega} K(x)|x|^{-\alpha p^{*}}\left(\left|w_{2}\right|^{p_{2}-1}+|u|^{p_{2}-1}\right)|v|^{q} \mathrm{~d} x\right] \\
\leq & 4^{r-1}\left[2^{p_{1}+1} a_{1}^{r}\left(\left\|w_{1}\right\|_{L_{K, \alpha}^{p_{1}-1}}^{p_{1}}+\|u\|_{L_{K, \alpha}^{p_{1}-1}}^{p_{1}}\right)\|v\|_{L_{K, \alpha}^{p_{1} q}}^{q}\right. \\
& \left.+2^{p_{2}+1} a_{2}^{r}\left(\left\|w_{2}\right\|_{L_{K, \alpha}^{p_{2}-1}}^{p_{2}}+\|u\|_{L_{K, \alpha}^{p_{2}}}^{p_{2}-1}\right)\|v\|_{L_{K, \alpha}^{p_{2} q}}^{q}\right] \\
< & +\infty .
\end{aligned}
$$

Since $u_{n} \rightarrow u$ for a.e. $x \in \Omega$, by (3.17), (3.18), and Lebesgue's dominated convergence theorem we have

$$
\lim _{i \rightarrow \infty} \int_{\Omega} K(x)|x|^{-\alpha p^{*}}\left|g\left(x, u_{k_{i}}\right)-g(x, u)\right|^{r}|v|^{q} \mathrm{~d} x=0
$$

which contradicts (3.16). Hence (3.15) holds.

Similarly, we can prove the following lemma.

Lemma 3.7 Let $p_{1}, p_{2}>1, r \geq 1$, and $\Omega \subseteq \mathbb{R}^{N}$. Let $g(x, t)$ be a Carathéodory function on $\Omega \times \mathbb{R}$ satisfying (3.14). If $u_{n} \rightarrow u$ in $L_{K, \alpha}^{p_{1}}(\Omega) \cap L_{K, \alpha}^{p_{2}}(\Omega)$ and $u_{n} \rightarrow u$ for a.e. $x \in \Omega$, then

$$
\lim _{n \rightarrow \infty} \int_{\Omega} K(x)|x|^{-\alpha p^{*}}\left|g\left(x, u_{n}\right)-g(x, u)\right|^{r}\left|u_{n}-u\right| d x=0
$$

Lemma 3.8 Under assumptions (VK1), $(V K 2),\left(f_{1}\right),\left(f_{2}\right)$, and $\left(f_{3}\right)$, any sequence $\left\{u_{n}\right\} \subset E$ satisfying (3.6) has a convergent subsequence in $E$.

Proof By Lemma 3.5 the sequence $\left\{u_{n}\right\}$ is bounded in $E$. Going if necessary to a subsequence, we can assume that $u_{n} \rightarrow u$ in $E$. By Lemma 3.4, $u_{n} \rightarrow u$ in $L_{K, \alpha}^{q}\left(\mathbb{R}^{N}\right)$ for $q \in\left[p, p^{*}\right)$, which, together with Lemma 3.7, yields

$$
\int_{\mathbb{R}^{N}} K(x)|x|^{-\alpha p^{*}}\left|f\left(x, u_{n}\right)-f(x, u)\right|\left|u_{n}-u\right| \mathrm{d} x \rightarrow 0, \quad n \rightarrow \infty .
$$


Observe that

$$
\begin{aligned}
\left\langle J^{\prime}\left(u_{n}\right)-J^{\prime}(u), u_{n}-u\right\rangle & \\
= & \int_{\mathbb{R}^{N}}\left(a\left(x, \nabla\left(u_{n}-u\right)\right) \nabla\left(u_{n}-u\right)+V(x)|x|^{-\alpha p^{*}}\left|u_{n}-u\right|^{p}\right) \mathrm{d} x \\
& -\int_{\mathbb{R}^{N}} K(x)|x|^{-\alpha p^{*}}\left[f\left(x, u_{n}\right)-f(x, u)\right]\left(u_{n}-u\right) \mathrm{d} x \\
\geq & \int_{\mathbb{R}^{N}}\left(c_{1}\left(1+|x|^{-\alpha p}\right) h_{1}(x)\left|\nabla\left(u_{n}-u\right)\right|^{p}+V(x)|x|^{-\alpha p^{*}}\left|u_{n}-u\right|^{p}\right) \mathrm{d} x \\
& -\int_{\mathbb{R}^{N}} K(x)|x|^{-\alpha p^{*}}\left[f\left(x, u_{n}\right)-f(x, u)\right]\left(u_{n}-u\right) \mathrm{d} x \\
\geq & k_{0} p\left\|u_{n}-u\right\|^{p}-\int_{\mathbb{R}^{N}} K(x)|x|^{-\alpha p^{*}}\left[f\left(x, u_{n}\right)-f(x, u)\right]\left(u_{n}-u\right) \mathrm{d} x .
\end{aligned}
$$

It is clear that

$$
\left\langle J^{\prime}\left(u_{n}\right)-J^{\prime}(u), u_{n}-u\right\rangle \rightarrow 0, \quad n \rightarrow \infty
$$

From (3.20)-(3.22) we have $\left\|u_{n}-u\right\| \rightarrow 0, n \rightarrow \infty$.

Lemma 3.9 Under assumptions (VK1), (VK2), $\left(f_{1}\right),\left(f_{2}\right)$, and $\left(f_{5}\right)$, any sequence $\left\{u_{n}\right\} \subset E$ satisfying (3.6) has a convergent subsequence in $E$.

Proof First, we prove that $\left\{u_{n}\right\}$ is bounded in $E$. To this end, by contradiction set $\left\|u_{n}\right\| \rightarrow$ $\infty$ as $n \rightarrow \infty$. Let $v_{n}=\frac{u_{n}}{\left\|u_{n}\right\|}$. Then $\left\|v_{n}\right\|=1$. By (2.2), (2.3), (3.2), $\left(A_{3}\right),\left(f_{5}\right)$, and $h_{1}(x) \geq 1$, for large $n \in \mathbb{N}$, we have

$$
\begin{aligned}
c^{*}+1 \geq & J\left(u_{n}\right)-\frac{1}{\mu}\left\langle J^{\prime}\left(u_{n}\right), u_{n}\right\rangle \\
\geq & \int_{\mathbb{R}^{N}}\left(A\left(x, \nabla u_{n}\right)+\frac{1}{p} V(x)|x|^{-\alpha p^{*}}\left|u_{n}\right|^{p}\right) \mathrm{d} x-\int_{\mathbb{R}^{N}} K(x)|x|^{-\alpha p^{*}} F\left(x, u_{n}\right) \mathrm{d} x \\
& -\frac{1}{\mu} \int_{\mathbb{R}^{N}}\left(p A\left(x, \nabla u_{n}\right)+V(x)|x|^{-\alpha p^{*}}\left|u_{n}\right|^{p}\right) \mathrm{d} x \\
& +\frac{1}{\mu} \int_{\mathbb{R}^{N}} K(x)|x|^{-\alpha p^{*}} f\left(x, u_{n}\right) u_{n} \mathrm{~d} x \\
\geq & \left(1-\frac{p}{\mu}\right) \int_{\mathbb{R}^{N}}\left(k_{0} h_{1}(x)\left(1+|x|^{-\alpha p}\right)\left|\nabla u_{n}\right|^{p}+\frac{1}{p} V(x)|x|^{-\alpha p^{*}}\left|u_{n}\right|^{p}\right) \mathrm{d} x \\
& -\int_{\mathbb{R}^{N}} K(x)|x|^{-\alpha p^{*}} F\left(x, u_{n}\right) \mathrm{d} x \\
& +\frac{1}{\mu} \int_{\mathbb{R}^{N}} K(x)|x|^{-\alpha p^{*}} f\left(x, u_{n}\right) u_{n} \mathrm{~d} x \\
\geq & \left(1-\frac{p}{\mu}\right) k_{0}\left\|u_{n}\right\|^{p}-\int_{\mathbb{R}^{N}} K(x)|x|^{-\alpha p^{*}}\left[\frac{u_{n}}{\mu} f\left(x, u_{n}\right)\right. \\
& \left.+\frac{\varrho}{\mu}\left|u_{n}\right|^{p}\right] \mathrm{d} x+\frac{u_{n}}{\mu} \int_{\mathbb{R}^{N}} K(x)|x|^{-\alpha p^{*}} f\left(x, u_{n}\right) \mathrm{d} x
\end{aligned}
$$




$$
\begin{aligned}
& =\left(1-\frac{p}{\mu}\right) k_{0}\left\|u_{n}\right\|^{p}-\frac{\varrho}{\mu} \int_{\mathbb{R}^{N}} K(x)|x|^{-\alpha p^{*}}\left|u_{n}\right|^{p} \mathrm{~d} x \\
& =\left(1-\frac{p}{\mu}\right) k_{0}\left\|u_{n}\right\|^{p}-\frac{\varrho}{\mu}\left\|u_{n}\right\|_{L_{K, \alpha}^{p}\left(\mathbb{R}^{N}\right)^{\prime}}
\end{aligned}
$$

which implies

$$
1 \leq \frac{\varrho}{(\mu-p) k_{0}} \limsup _{n \rightarrow \infty}\left\|v_{n}\right\|_{L_{K, \alpha}^{p}}^{p}\left(\mathbb{R}^{N}\right)
$$

Passing to a subsequence, we may assume that $v_{n} \rightarrow v$ in $E$. Then by Lemma 3.4, $E$ is compactly embedded in $L_{K, \alpha}^{q}\left(\mathbb{R}^{N}\right), q \in\left[p, p^{*}\right), v_{n} \rightarrow v$ in $L_{K, \alpha}^{q}\left(\mathbb{R}^{N}\right), q \in\left[p, p^{*}\right)$, and $v_{n} \rightarrow v$ a.e. on $\mathbb{R}^{N}$. Hence from (3.23) it follows that $v \neq 0$. By a similar fashion as for (3.13), we can get a contradiction. Thus $\left\{u_{n}\right\}$ is bounded in $E$. The rest of the proof is the same as that in Lemma 3.8.

Lemma 3.10 Under assumptions (VK1), (VK2), $\left(f_{1}\right)$, and $\left(f_{2}\right)$, for any finite-dimensional subspace $\tilde{E} \subset E$, we have

$$
J(u) \rightarrow-\infty \quad \text { as }\|u\| \rightarrow \infty, u \in \tilde{E} .
$$

Proof Arguing indirectly, assume that for some sequence $\left\{u_{n}\right\} \subset \tilde{E}$ with $\left\|u_{n}\right\| \rightarrow \infty, n \rightarrow$ $\infty$, there exists $M>0$ such that $J\left(u_{n}\right) \geq-M$ for all $n \in \mathbb{N}$. Set $v_{n}=\frac{u_{n}}{\left\|u_{n}\right\|}$; then $\left\|v_{n}\right\|=1$. Passing to a subsequence, we may assume that $v_{n} \rightarrow v$ in $E$. Since $\tilde{E}$ is finite dimensional, $v_{n} \rightarrow v \in \tilde{E}$ in $E, v_{n} \rightarrow v$ a.e. on $\mathbb{R}^{N}$, and thus $\|v\|=1$. Hence we can get a contradiction by a similar fashion as for (3.13).

Corollary 3.11 Under assumptions (VK1), (VK2), $\left(f_{1}\right)$, and $\left(f_{2}\right)$, for any finite-dimensional subspace $\tilde{E} \subset E$, there is $R=R(\tilde{E})>0$ such that

$$
J(u) \leq 0, \quad \forall u \in \tilde{E},\|u\| \geq R .
$$

Since $E$ is a reflexive separable Banach space, there exist $\left\{e_{n}\right\}_{n=1}^{\infty} \subset E$ and $\left\{e_{n}^{*}\right\}_{n=1}^{\infty} \subset E^{*}$ such that

$$
\begin{aligned}
& \left\langle e_{n}^{*}, e_{m}\right\rangle= \begin{cases}1 & \text { if } n=m, \\
0 & \text { if } n \neq m,\end{cases} \\
& E=\overline{\operatorname{span}}\left\{e_{n}: n=1,2, \ldots\right\}, \text { and } E^{*}=\overline{\operatorname{span}}\left\{e_{n}^{*}: n=1,2, \ldots\right\} .
\end{aligned}
$$

For $k=1,2, \ldots$, we denote

$$
X_{k}=\overline{\operatorname{span}}\left\{e_{k}\right\}, \quad Y_{k}=\bigoplus_{j=1}^{k} X_{j} \quad \text { and } \quad Z_{k}=\overline{\bigoplus_{j=k+1}^{\infty} X_{j}} .
$$

Lemma 3.12 Let (VK1) and (VK2) be satisfied. Then for any $p \leq q<p^{*}$, we have

$$
\eta_{k}(q):=\sup _{u \in Z_{k},\|u\|=1}\|u\|_{L_{K, \alpha}^{q}\left(\mathbb{R}^{N}\right)} \rightarrow 0, \quad k \rightarrow \infty .
$$


Proof It is clear that $0 \leq \eta_{k+1} \leq \eta_{k}$, so that $\eta_{k} \rightarrow \eta \geq 0(k \rightarrow \infty)$. For every $k \in \mathbb{N}$, there exists $u_{k} \in Z_{k}$ satisfying

$$
\left\|u_{k}\right\|=1, \quad 0 \leq \eta_{k}-\left\|u_{k}\right\|_{L_{K, \alpha}^{q}\left(\mathbb{R}^{N}\right)}<\frac{1}{k} .
$$

Then there exists a subsequence of $\left\{u_{k}\right\}$ (which we still denote by $u_{k}$ ) such that $u_{k} \rightarrow u$ and

$$
\left\langle e_{n}^{*}, u\right\rangle=\lim _{k \rightarrow \infty}\left\langle e_{n}^{*}, u_{k}\right\rangle=0, \quad n=1,2, \ldots
$$

which implies that $u=0$, and so $u_{k} \rightarrow 0$. By the compact embedding $E \hookrightarrow L_{K, \alpha}^{q}\left(\mathbb{R}^{N}\right)$ we have $u_{k} \rightarrow 0$ in $L_{K, \alpha}^{q}\left(\mathbb{R}^{N}\right)$. Hence we get $\eta_{k} \rightarrow 0$.

By Lemma 3.12 we can choose an integer $m \geq 1$ such that

$$
\|u\|_{L_{K, \alpha}^{p}\left(\mathbb{R}^{N}\right)}^{p} \leq \frac{p k_{0}}{2 c_{1}}\|u\|^{p}, \quad\|u\|_{L_{K, \alpha}^{\beta}\left(\mathbb{R}^{N}\right)}^{\beta} \leq \frac{\beta k_{0}}{4 c_{2}}\|u\|^{\beta}, \quad \forall u \in Z_{m} .
$$

Lemma 3.13 Let (VK1), (VK2), and $\left(f_{1}\right)$ be satisfied. Then there exist constants $\rho, \theta>0$ such that $\left.J\right|_{\partial B_{\rho} \cap Z_{m}} \geq \theta$.

Proof From (2.2), (2.4), (3.2), (3.24), and $h_{1}(x) \geq 1$, for $u \in Z_{m}$, choosing $\rho:=\|u\|=\frac{1}{2}$, we get

$$
\begin{aligned}
J(u)= & \int_{\mathbb{R}^{N}}\left(A(x, \nabla u)+\frac{1}{p} V(x)|x|^{-\alpha p^{*}}|u|^{p}\right) \mathrm{d} x-\int_{\mathbb{R}^{N}} K(x)|x|^{-\alpha p^{*}} F(x, u) \mathrm{d} x \\
\geq & \int_{\mathbb{R}^{N}}\left(k_{0} h_{1}(x)\left(1+|x|^{-\alpha p}\right)|\nabla u|^{p}+\frac{1}{p} V(x)|x|^{-\alpha p^{*}}|u|^{p}\right) \mathrm{d} x \\
& -\int_{\mathbb{R}^{N}} K(x)|x|^{-\alpha p^{*}} F(x, u) \mathrm{d} x \\
\geq & k_{0}\|u\|^{p}-\int_{\mathbb{R}^{N}} K(x)|x|^{-\alpha p^{*}}\left(\frac{c_{1}}{p}|u|^{p}+\frac{c_{2}}{\beta}|u|^{\beta}\right) \mathrm{d} x \\
= & k_{0}\|u\|^{p}-\frac{c_{1}}{p}\|u\|_{L_{K, \alpha}^{p}\left(\mathbb{R}^{N}\right)}^{p}-\frac{c_{2}}{\beta}\|u\|_{L_{K, \alpha}^{\beta}}^{\beta}\left(\mathbb{R}^{N}\right) \\
\geq & \frac{k_{0}}{2}\|u\|^{p}-\frac{k_{0}}{4}\|u\|^{\beta} \\
= & \frac{k_{0} \cdot 2^{\beta-p+1}-k_{0}}{2^{\beta+2}}:=\theta>0 .
\end{aligned}
$$

This completes the proof.

Proof of Theorem 1.4 Let $X=E, Y=Y_{m}$, and $Z=Z_{m}$. By Lemma 3.5, Lemma 3.8, Lemma 3.13, and Corollary 3.11 all conditions of Lemma 3.1 are satisfied. Thus problem (1.1) possesses infinitely many nontrivial solutions.

Proof of Theorem 1.5 Let $X=E, Y=Y_{m}$, and $Z=Z_{m}$. Obviously, the rest of the proof is the same as that of Theorem 1.4 by using Lemma 3.9 instead of Lemmas 3.5 and 3.8. 


\section{Acknowledgements}

The authors would like to thank the handling editors and the anonymous reviewers.

\section{Funding}

This work was supported by National Natural Science Foundation of China (Grant Nos. 11661053, 11771198, 11961045, and 11901276), the Provincial National Natural Science Foundation of Jiangxi (Grant Nos. 20161BAB201009, 20181BAB201003, 20202BAB201001, and 20202BAB211004).

\section{Availability of data and materials}

Not applicable.

\section{Competing interests}

The authors declare that they have no competing interests.

\section{Authors' contributions}

The authors conceived of the study, drafted the manuscript, and approved the final manuscript.

\section{Publisher's Note}

Springer Nature remains neutral with regard to jurisdictional claims in published maps and institutional affiliations.

\section{Received: 7 September 2020 Accepted: 12 April 2021 Published online: 26 April 2021}

\section{References}

1. Alves, C.O., Soares, S.H.M.: Existence and concentration of positive solutions for a class of gradient systems. Nonlinear Differ. Equ. Appl. 12, 437-457 (2006)

2. Alves, C.O., Souto, M.A.S.: Existence of solutions for a class of elliptic equations in $\mathbb{R}^{N}$ with vanishing potentials. J. Differ. Equ. 252, 5555-5568 (2012)

3. Alves, C.O., Souto, M.A.S.: Existence of solutions for a class of nonlinear Schrödinger equations with potential vanishing at infinity. J. Differ. Equ. 254, 1977-1991 (2013)

4. Alves, C.O., Souto, M.A.S., Montenegro, M.: Existence of solution for two classes of elliptic problems in $\mathbb{R}^{N}$ with zero mass. J. Differ. Equ. 252, 5735-5750 (2012)

5. Ambrosetti, A., Felli, V., Malchiodi, A.: Ground states of nonlinear Schrödinger equations with potentials vanishing at infinity. J. Eur. Math. Soc. 7, 117-144 (2005)

6. Bartolo, T., Benci, V., Fortunato, D.: Abstract critical point theorems and applications to some nonlinear problems with strong resonance at infinity. J. Nonlinear Anal. 7, 241-273 (1983)

7. Bastos, W.D., Miyagaki, O.H., Vieira, R.S.: Existence of solutions for a class of degenerate quasilinear equation in $\mathbb{R}^{N}$ with vanishing potentials. Bound. Value Probl. 2013, 92 (2013)

8. Bastos, W.D., Miyagaki, O.H., Vieira, R.S.: Positive solution for a class of degenerate quasilinear elliptic equations. Milan J. Math. 82, 213-231 (2014)

9. Benci, V., Crisant, C.R., Micheletti, A.M.: Existence of solutions of nonlinear Schrödinger equations with $V(\infty)=0$. Prog. Nonlinear Differ. Equ. Appl. 66, 53-65 (2005)

10. Berestycki, H., Lions, P.L.: Nonlinear scalar field equations, I existence of a ground state. Arch. Ration. Mech. Anal. 82, 313-345 (1983)

11. Byeon, J., Wang, Z.Q.: Spherical semiclassical states of a critical frequency for Schrödinger equations with decaying potentials. J. Eur. Math. Soc. 8, 217-228 (2006)

12. Byeon, J., Wang, Z.Q.: Standing waves for nonlinear Schrödinger equations with singular potentials. Nonlinear Anal. 26, 943-958 (2009)

13. Caffarelli, L., Kohn, R., Nirenberg, L.: First order interpolations inequalities with weights. Compos. Math. 53, 259-275 (1984)

14. Catrina, F., Wang, Z.Q.: On the Caffarelli-Kohn-Nirenberg inequalities: sharp constants. Existence (and nonexistence) and symmetry of extremal functions. Commun. Pure Appl. Math. 54, 229-258 (2001)

15. Chen, J.H., Huang, X.J., Cheng, B.T., Luo, H.X.: Existence and multiplicity of nontrivial solutions for nonlinear Schrödinger equations with unbounded potentials. Filomat 32, 2465-2481 (2018)

16. Diaz, J..: Nonlinear partial differential equations and free boundaries. J. Appl. Math. Mech. (1985)

17. Duc, D.M., Vu, N.T.: Nonuniformly elliptic equations of p-Laplacian type. Nonlinear Anal. 61, 1483-1495 (2005)

18. Kryszewski, W., Szulkin, A.: Generalized linking theorem with an application to semilinear Schrödinger equation. Adv. Differ. Equ. 3, 441-472 (1998)

19. Lin, X.Y., Tang, X.H.: Existence of infinitely many solutions for $\mathrm{p}$-Laplacian equations in $\mathbb{R}^{N}$. Nonlinear Anal. 92, 72-81 (2013)

20. Miyagaki, O.H., Santana, C.R., Vieira, R.S.: Ground states of degenerate quasilinear Schrödinger equation with vanishing potentials. J. Nonlinear Anal. 189, 111587 (2019)

21. Pino, M.D., Felmer, P.: Local mountain passes for semilinear elliptic problems in unbounded domains. Calc. Var. Partial Differ. Equ. 4, 121-137 (1996)

22. Rabinowitz, P.H.: Minimax Methods in Critical Point Theory with Applications to Differential Equations. Am. Math. Soc., Providence (1986)

23. Rabinowitz, P.H.: On a class of nonlinear Schrödinger equations. Z. Angew. Math. Phys. 43, 270-291 (1992)

24. Tang, X.H.: Infinitely many solutions for semilinear Schrödinger equations with sign-changing potential and nonlinearity. J. Math. Anal. Appl. 401, 407-415 (2013)

25. Wang, Z.Q., Willem, M.: Singular minimization problems. J. Differ. Equ. 2, 307-320 (2000) 